\title{
Модель генерации флуоресцентного сигнала интеркаляционного красителя в ходе полимеразной цепной реакции
}

\author{
(C) А.А. Федоров, ${ }^{1}$ Д.Г. Сочивко, ${ }^{2}$ Д.А. Варламов ${ }^{2}$ \\ ${ }^{1}$ Институт аналитического приборостроения РАН, \\ 190103 Санкт-Петербург, Россия \\ 23АО „Синтол“, \\ 127550 Москва, Россия \\ e-mail: f_aa@mail.ru \\ Поступило в Редакцию 19 декабря 2019 г. \\ В окончательной редакции 25 февраля 2020 г. \\ Принято к публикации 3 марта 2020 г.
}

В настоящее время предложен ряд моделей полимеразной цепной реакции, имеющих своей целью получение точных количественных оценок результатов реакции. В таких моделях обычно отождествляют кинетику продукта реакции и используемого флуоресцентного репортера. Предложена модель полимеразной цепной реакции, результаты которой регистрируют с помощью интеркаляционного красителя. Анализ результатов моделирования демонстрирует заметные различия между кинетикой продукта реакции и флуоресцентным сигналом связанного интеркалятора.

Ключевые слова: модель полимеразной цепной реакции, кинетическая кривая, интеркалирующий краситель.

DOI: 10.21883/JTF.2020.09.49693.422-19

\section{Введение}

Полимеразная цепная реакция (ПЦР) является ключевым молекулярно-генетическим методом, применяемым для решения широкого круга научных и практических задач выявления и количественного анализа специфических последовательностей ДНК. В основе метода лежит амплификация целевого фрагмента ДНК, т.е. многократное циклическое копирование определенного участка ДНК, выделяемого с помощью фланкирующих одноцепочечных олигонуклеотидов - праймеров. На каждом цикле реакции происходит удвоение количества амплифицируемых фрагментов.

Первоначально ПЦР не использовали как количественный метод. Длительное время учет результатов ПЦР проводили по окончании реакции с помощью гельэлектрофореза, анализируя наличие фрагментов заданной длины. Позднее было предложено усовершенствовать технологию ПЦР за счет регистрации продуктов реакции непосредственно в процессе ее проведения, в результате чего появился метод ПЦР в реальном времени (ПЦР-PВ; real-time PCR) [1].

В работе [1] было продемонстрировано, что постановка ПЦР в присутствии известного интеркалирующего красителя ДНК, бромистого этидия, позволяет проследить накопление копий амплифицируемого фрагмента на каждом цикле. Это позволяет построить кинетическую кривую процесса [2], имеющую сигмоидный характер с начальным фоновым участком, участком экспоненциального роста, переходящим в фазу насыщения. Чем больше исходных фрагментов вносят в реакцию, тем раньше ее кинетическая кривая отрывается от фонового уровня, при этом разница в 1 цикл реакции соответствует 2-кратной разнице в начальной концентрации фрагмента. Для количественного анализа обычно применяют контрольные образцы с известной концентрацией целевого фрагмента ДНК, относительно которых вычисляют концентрацию исследуемых образцов.

Интеркаляция - обратимое включение молекулы или группы между другими молекулами или группами. Интеркалирующие красители (ИК) это группа флуоресцентных органических соединений, способных к нековалентному связыванию с одно- и/или двуцепочечной ДНК, при этом связывание с ДНК приводит к значительному усилению флуоресценции. В настоящее время широко применяется краситель SYBR Green I с длиной волны эмиссии $520 \mathrm{~nm}$, флуоресценция которого возрастает более чем в 100 раз в результате связывания с двуцепочечной ДНК. Данный краситель часто применяют для детекции продуктов в ходе ПЦР-РВ. В высоких концентрациях SYBR Green I ингибирует ДНК-полимеразу, в результате снижается эффективность амплификации.

Основным недостатком использования ИК для ПЦР-РВ является способность связываться с любой двуцепочечной ДНК, появляющейся в реакционной смеси, в результате чего флуоресцентный сигнал может генерироваться как целевым продуктом ПЦР, так и неспецифическими продуктами побочных реакций. Поэтому для получения корректных результатов обычно дополнительно подтверждают наличие целевого продукта амплификации с помощью температурной денатурации („плавления“) ампликонов. В связи с возможностью ошибочных результатов ПЦР-РВ с интеркалирующими красителями, несмотря на дешевизну и удобство, в 
практической ДНК-диагностике используют с осторожностью.

Альтернативным способом детекции продуктов ПЦРРВ является использование специфических одноцепочечных олигонуклеотидов, комплементарных анализируемой ДНК-мишени, называемых гибридизационными зондами. На концах зонда ковалентно присоединены молекулы флуоресцентного красителя и тушителя флуоресценции, в результате чего в растворе зонд флуоресцирует слабо. В процессе работы ДНК-полимеразы зонд, связавшийся с амплифицируемым фрагментом, расщепляется за счет экзонуклеазной активности фермента, тушитель и краситель высвобождаются, и флуоресценция красителя многократно возрастает. Поскольку последовательность зонда может связаться только с целевым фрагментом, специфичность детекции продуктов ПЦРРВ становится практически абсолютной.

В силу различных механизмов работы гибридизационных зондов и ИК, кинетические кривые ПЦР-РВ, полученные с их помощью, будут различаться. Это различие необходимо учитывать при разработке математических методов анализа ПЦР-РВ, а также математических моделей реакции.

Предложенные в опубликованных работах модели ПЦР позволяют генерировать кинетические кривые ПЦР-РВ как функции накопления копий целевого фрагмента ДНК. При оценке работы моделей обычно сопоставляют такие кривые с экспериментальными данными. Однако представление об эквивалентности экспериментальных и теоретических кривых представляется нам не совсем обоснованным, тем более что для получения экспериментальных кривых используются как гибридизационные зонды [3], так и ИК, в основном SYBR Green [4-7]. В случае, когда фактор флуоресцентной детекции оговаривается, авторы используют простую линейную зависимость для взаимного пересчета этих величин $[6,7]$.

Ранее нами была предложена модель ПЦР-РВ, в которой флуоресцентный сигнал генерировался гибридизационным зондом [8]. Модель показала различие кривых продукта ПЦР и флуоресцентного сигнала, а также позволила с высокой точностью аппроксимировать экспериментальные кинетические кривые ПЦР-РВ.

В настоящей работе представлена модель ПЦР-РВ, в которой для генерации флуоресцентного сигнала использован интеркалирующий краситель. Предлагаемая модель учитывает основные компоненты и стадии процесса ПЦР, происходящие в течение одного цикла ПЦР.

\section{1. Модель формирования флуоресцентного сигнала интекалирующего красителя}

На первой стадии каждого цикла ПЦР реакционная смесь нагревается до $95^{\circ} \mathrm{C}$, в результате чего происходит денатурация двухцепочной ДНК $\left(F \| F^{\prime}\right)$ на две комплементарные одноцепочные молекулы - F и $\mathrm{F}^{\prime}$. Далее смесь охлаждается, наступает стадия отжига, на которой специфические к $5^{\prime}$-концевому участку каждой из цепей праймеры $\operatorname{Pr}$ и $\operatorname{Pr}^{\prime}$ имеют возможность гибридизоваться с цепями $F$ и $F^{\prime}$. Фермент ДНК-полимераза связывается с комплексом праймера и цепи. Далее наступает черед стадии элонгации, на которой фермент начинает достраивать новую, комплементарную цепь, используя нуклеотиды реакционной смеси. В случае успешного завершения синтеза одноцепочная молекула ДНК $(F$ или $\left.F^{\prime}\right)$ превращается в двухцепочную молекулу - копию исходной $\left(F \| F^{\prime}\right)$.

Две исходные цепи $F$ и $F^{\prime}$ могут соединиться обратно в исходную двойную цепь, даже если с одной из них или с обеими успели связаться праймеры, но элонгация еще не началась. Данная реакция принимается за необратимую, поскольку при типичной температуре элонгации диссоциация таких комплексов практически не происходит. Интеркалирующий краситель $(I)$, связываясь со всеми двойными цепями, присутствующими в реакции, начинает генерировать сигнал, интенсивность которого предполагается пропорциональной количеству связанного ИК. Далее смесь опять нагревают для денатурации ДНК в реакционной смеси, и начинается следующий цикл.

Введем следующие обозначения промежуточных комплексов данной реакции: $S$ и $S^{\prime}$ - комплексы фрагмента ДНК и соответствующего праймера $(S=F+\operatorname{Pr}$, $\left.S^{\prime}=F^{\prime}+\operatorname{Pr}^{\prime}\right) ; I-$ молекула ИК.

Bсе промежуточные комплексы в случае неучастия в синтезе комплементарной цепи диссоциируют при нагревании на исходные компоненты. Для продуктов реакции введем такие обозначения: $P_{1}$ и $P_{1}^{\prime}-$ продукт двойная цепь, полученная синтезом и не внесшая вклад во флуоресцентный отклик системы; $P_{2}$ - двойная цепь, полученная гибридизацией одиночных.

С точки зрения результата ПЦР, все перечисленные продукты не различаются между собой, поскольку являются просто двойными цепями $F \| F^{\prime}$.

С помощью принятых обозначений схему реакций описанного процесса можно представить так:

первичные реакции

$$
\begin{gathered}
F+\operatorname{Pr} \underset{k_{-1}}{\stackrel{k_{1}}{\longrightarrow}} S, F^{\prime}+\operatorname{Pr}^{\prime} \underset{k_{-1}}{\stackrel{k_{1}}{\longrightarrow}} S^{\prime}, \\
F+F^{\prime} \stackrel{k_{2}}{\longrightarrow} P_{2} ;
\end{gathered}
$$

вторичные реакции

$$
\begin{gathered}
F+S^{\prime} \stackrel{k_{2}}{\longrightarrow} P_{2}+\operatorname{Pr}^{\prime}, F^{\prime}+S \stackrel{k_{2}}{\longrightarrow} P_{2}+\operatorname{Pr}, \\
S+S^{\prime} \stackrel{k_{2}}{\longrightarrow} P_{2}+\operatorname{Pr}^{\prime}+\operatorname{Pr}
\end{gathered}
$$

реакции синтеза продукта

$$
S \stackrel{k_{m}}{\longrightarrow} P_{1}, S^{\prime} \stackrel{k_{m}}{\longrightarrow} P_{1}^{\prime}
$$


реакции связывания ИК

$$
\begin{gathered}
I_{\text {free }}+P_{1} \stackrel{k_{I C}}{\longrightarrow} I_{\text {bound }}, I_{\text {free }}+P_{2} \stackrel{k_{I C}}{\longrightarrow} I_{\text {bound }}, \\
I_{\text {free }}+P_{1}^{\prime} \stackrel{k_{I C}}{\longrightarrow} I_{\text {bound }} .
\end{gathered}
$$

Константа скорости ферментативного синтеза цепей $k_{M}$ может быть представлена в виде $k_{M}=V_{\max } / K_{M}$, где $V_{\max }=1 \cdot 10^{-8} \mathrm{M} \cdot \mathrm{s}^{-1}$, а $K_{M}=2 \cdot 10^{-9} \mathrm{M}[9,10]$.

Важным вопросом является описание работы ИК. Особенностью работы ИК является, во-первых, отсутствие определенного места связывания с ДНК (краситель связывается с любым участком двойной цепи). Во-вторых, поскольку молекула ИК существенно меньше, чем ДНК, и участок связывания по размеру соответствует примерно 5 нуклеотидам, очевидно, что на одну ДНК может сесть более одной молекулы ИК. Учитывая, что общая длина выделяемого праймерами и синтезируемого в ходе ПЦР фрагмента ДНК составляет около 100-150 нуклеотидов, теоретический предел связывания ИК к ДНК составляет 30 к 1 . Исходя из этих фактов, будем рассматривать $k_{I C}$ не как параметр, а как функцию, для которой введем следующее уравнение, описывающее превращение свободного ИК $I_{\text {free }}$ в связанный $I_{\text {bound }}$.

$$
\begin{aligned}
& k_{I C}= \\
& = \begin{cases}0, & I_{\text {bound }}>\Omega\left(P_{1}+P_{2}+P_{1}^{\prime}\right) \\
k_{1} \cdot\left(1-\frac{I_{\text {bound }}}{\Omega\left(P_{1}+P_{2}+P_{1}^{\prime}\right)},\right. & \left.I_{\text {bound }} \leq \Omega\left(P_{1}+P_{2}+P_{1}^{\prime}\right)\right),\end{cases}
\end{aligned}
$$

где $\Omega$ - максимальное соотношение количества связанного ИК и двойных цепей. Поскольку данный параметр заранее неизвестен, его значение представляется целесообразным подобрать на этапе сравнения модельных ПЦР-РВ кривых с экспериментальными.

Будем считать, что процессы синтеза ДНК на обеих комплементарных цепях полностью идентичны, т.е. все концентрации комплементарных одноцепочечных фрагментов, все соответствующие константы ассоциации и диссоциации совпадают. С точки зрения модельной системы это означает возможность замены концентраций, содержащих штрихованные компоненты, на соответствующие нештрихованные: $C_{F}(t)$ на $C_{F^{\prime}}(t), C_{S}(t)$ на $C_{S^{\prime}}(t)$ и т.д. В этом случае система модельных уравнений может быть несколько упрощена и представлена в виде

$$
\begin{gathered}
\frac{d C_{F}}{d t}=-k_{1} C_{F} C_{\operatorname{Pr}}+k_{-1} C_{S}-k_{2} C_{F} C_{F}-k_{2} C_{S} C_{F} \\
\frac{d C_{\operatorname{Pr}}}{d t}=-k_{1} C_{F} C_{\operatorname{Pr}}+k_{-1} C_{S}+k_{2} C_{S} C_{F}+k_{2} C_{S} C_{S} \\
\frac{d C_{S}}{d t}=k_{1} C_{F} C_{\operatorname{Pr}}-k_{-1} C_{S}-k_{2} C_{S} C_{F}-k_{2} C_{S} C_{S}-k_{M} C_{S} \\
\frac{d C_{P_{2}}}{d t}=k_{2} C_{F} C_{F}+2 k_{2} C_{S} C_{F}+k_{2} C_{S} C_{S} \\
\frac{d C_{P_{1}}}{d t}=k_{M} C_{S} \\
\frac{d C_{I}}{d t}=k_{I C}\left(C_{P_{2}}+2 \cdot C_{P_{1}}\right)\left(C_{I}^{0}-C_{I}\right)
\end{gathered}
$$

где $C_{A}-$ концентрация компонента $A, C_{I}^{0}-$ общая концентрация ИК.

Реализация предложенной модели была выполнена в виде программы на языкеС ++ . Система (2) решалась численно методом Рунге-Кутта 4-го порядка. Программа позволяла повторять модельные вычисления заданное количество раз, имитируя необходимое количество последовательных циклов реакции. Для первого цикла ПЦР задавали начальную концентрацию цепей $C_{F}(0)$, праймеров $C_{\mathrm{Pr}}(0)$, ИК $C_{I}(0)$ и длительность этапа синтеза ДНК $T$.

В конце цикла определялась концентрация синтезированных ДНК $C_{P_{1}}(T)$ Для следующего цикла количество исходной ДНК увеличивалось на величину $C_{P_{1}}(T)$, на эту же величину уменьшалась начальная концентрация праймера для следующего цикла. В ходе этапа элонгации свободный ИК превращался в связанный по формуле (1). Количество связанного ИК на момент окончания цикла давало точку модельной кривой ПЦР-РВ. По окончании цикла весь связанный ИК снова переходил в свободную форму, т.е. количество ИК не менялось в течение модельного эксперимента.

\section{2. Результаты и выводы}

Экспериментальные ПЦР-РВ кривые были получены с использованием тест-системы для ПЦР-РВ анализа гена Fc фрагмента IgG рецептора IIIa человека (FCGR3A, GenBank NG_009066.1) производства ЗАО Синтол (г. Москва). Последовательность прямого праймера: CACATATTTACAGAATGGCAAAGG, обратного праймера: CTGAAGACACATTTTTACTCCCAА. Концентрация каждого праймера в реакционной смеси $C_{P \mathrm{r}}(0)=200 \mathrm{nM}$, фермента -5 units, количество ДНК гена Fc в модельных образцах составляло $10^{7}$ молекул ДНК на реакцию. В качестве ИК использовался SYBR GREEN I в концентрации $200 \mathrm{nM}$. Объем реакционной смеси - $25 \mu 1$. Эксперимент был поставлен на амплификаторе CFX96 (Bio-Rad, CША). Параметры термоциклирования: предварительный нагрев до $95^{\circ} \mathrm{C}-$ $120 \mathrm{~s}$, далее 50 циклов: $62^{\circ} \mathrm{C}-30 \mathrm{~s}, 95^{\circ} \mathrm{C}-15 \mathrm{~s}$. Для модельных образцов было проведено пять дублирующих экспериментов и получено пять экспериментальных кривых ПЦР-РВ. Экспериментальные кривые хорошо воспроизводились, для нормированных на максимум ПЦР-РВ кривых дисперсия значений флуоресценции на каждом цикле не превышала $0.3 \%$. На основании полученных данных была построена усредненная кривая ПЦР-РВ, которая и использовалась для сопоставления с результатами моделирования.

Результаты расчетов динамики интенсивности флуоресцентного сигнала по предложенной модели, а также соответствующая экспериментальная кривая ПЦР-РВ представлены на рис. 1. Видно, что модельная кривая хорошо аппроксимирует экспериментальную кривую ПЦР-РВ. Модель позволяет воспроизвести кинетику 
начального участка роста кривой, что важно для практического использования модели при анализе результатов реакции.

На рис. 2 показано влияние параметра $\Omega$ на точность аппроксимации. Как видно, наибольшая точность аппроксимации соответствует параметру $\Omega=1$, что соответствует начальным условиям реакции, поскольку концентрация ИК равна концентрации праймеров, и максимальное отношение связанного ИК к количеству двойных цепей равно единицы.

На рис. 3 сопоставляется модельные кинетические кривые по флуоресцентному сигналу и накоплению копий ДНК. Как видно из представленных данных, кинетика этих кривых существенно различается: кривая флуоресцентного сигнала отстает от кривой накопления копий почти на 1 цикл. Если использовать аппрок-

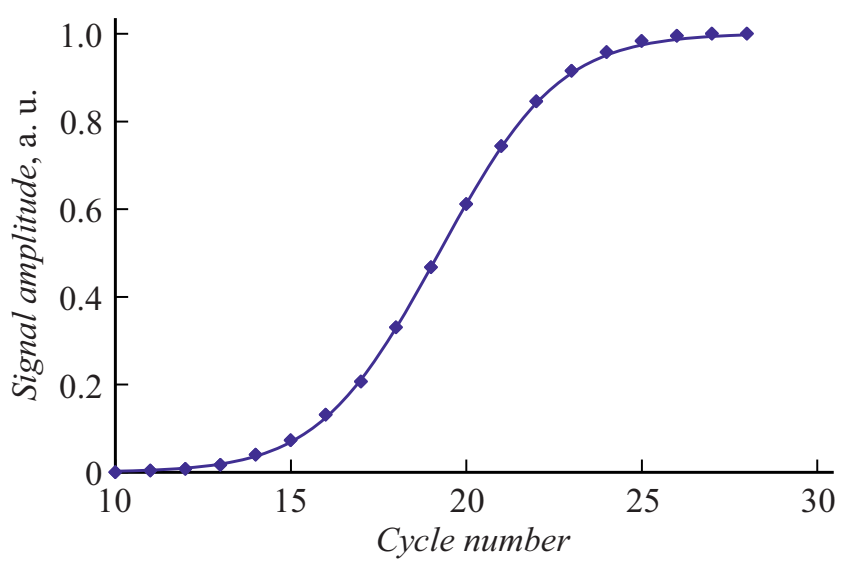

Рис. 1. Экспериментальная (сплошная линия) и модельная (ромбы) ПЦР-РВ кривые для $10^{7}$ молекул ДНК в анализируемой пробе. Модельная кривая отражает количество связанного ИК на данном цикле реакции. Параметр $\Omega=1$. По вертикальной оси отложены нормированные к максимуму значения функций.

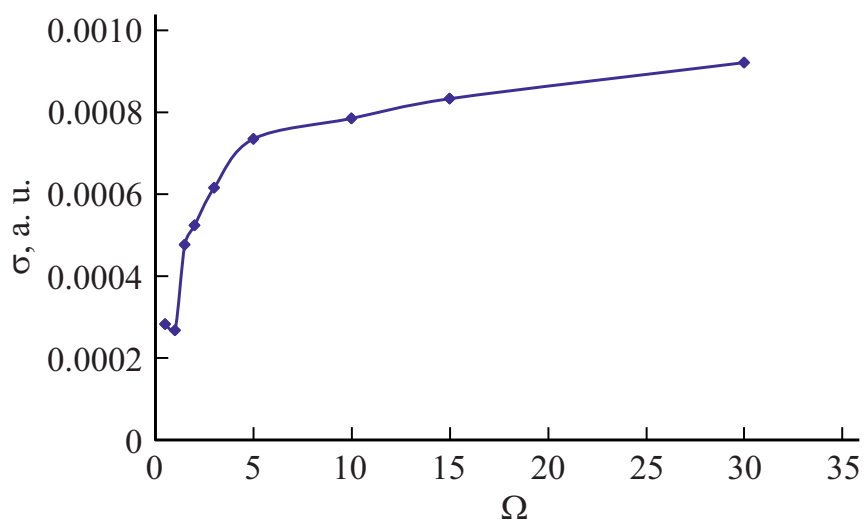

Рис. 2. Точность аппроксимации модельными кривыми ПЦР$\mathrm{PB}$, соответствующими различным значениям параметра $\Omega$, экспериментальной кривой для $10^{7}$ молекул ДНК в пробе. В качестве параметра точности аппроксимации $\sigma$ использована сумма разности квадратов значений модельной и экспериментальной ПЦР-РВ кривой.

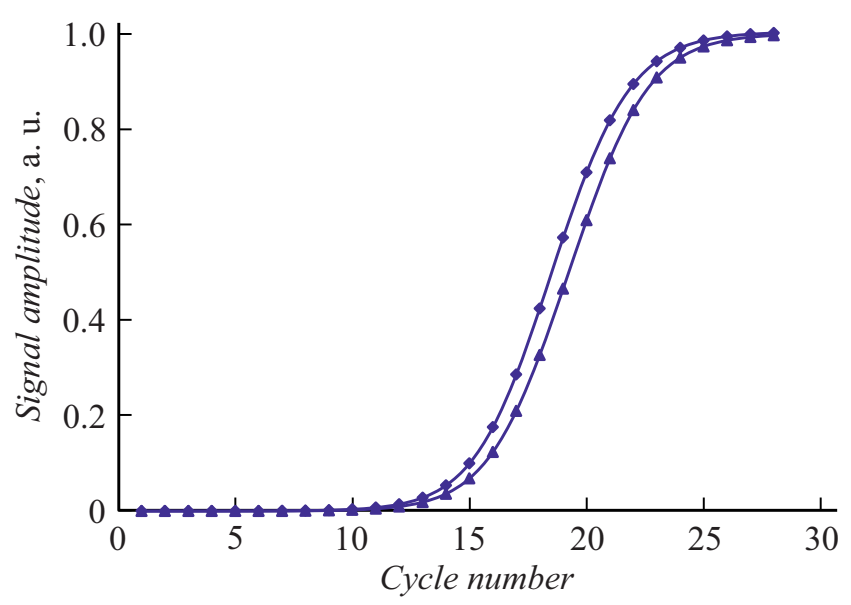

Рис. 3. Модельные кумулятивные кривые накопления копий исходной ДНК (ромбы) и связанного интеркаляционного красителя (треугольники) в ходе ПЦР-РВ. Функции нормированы на максимальные значения.

симацию флуоресцентного сигнала модельной кривой для анализа концентрации фрагментов в образце, то концентрация, полученная на основе модели, учитывающей только количество фрагментов, будет почти в два раза отличаться от реальной. В этой связи при аппроксимации экспериментальных данных, полученных с использованием ИК, следует применять специальную модель, отражающую динамику флуоресцентного сигнала ИК.

Представленные результаты демонстрируют высокую точность аппроксимации экспериментальных кривых ПЦР-РВ, что свидетельствует об адекватности предложенной модели. Важным результатом, полученным с помощью модели, является демонстрация того, что кривые накопления ДНК и сигнала связанного ИК не совпадают и не могут быть получены простым линейным пересчетом для всего диапазона ПЦР-РВ кривой. Следует отметить, что точность аппроксимации несколько ниже, чем для ранее опубликованной модели с зондом [8], что можно объяснить рядом не учтенных в модели эффектов, таких как кооперативность связывания ИК, ингибирование ИК самой реакции и „выгорание“ красителя.

\section{Финансирование работы}

Работа выполнена в ИАП РАН в рамках Государственного задания 075-00780-19-02 по теме № 0074-2019-0013 Министерства науки и высшего образования РФ.

\section{Конфликт интересов}

Авторы заявляют, что у них нет конфликта интересов. 


\section{Список литературы}

[1] Higuchi R., Dollinger G., Walsh S., Griffith R. // Biotechnology 1992. Vol. 10. P. 413-417.

[2] Higuchi R., Fockler C., Dollinger G., Watson R. // Biotechnology. 1993. Vol. 11. P. 1026-1030.

[3] Smith M.V., Miller C.R., Kohn M., Walker N.J., Portier C.J. // BMC Bioinformatics. 2007. Vol. 8. N 1. P. 409.

[4] Jones M.E., Mayne G.C., Wang T., Watson D.I., Hussey D.J. // BMC Bioinformatics. 2014. Vol. 15. P. 372.

[5] Lievens A., Van Aelst S., Van den Bulcke M., Goetghebeur E. // PLoS One. 2012. Vol. 11. P. e47112.

[6] Rutledge R.G., Stewart D. // PLoS One. 2010. Vol. 3. P. e9731.

[7] Liu W., Saint D.A. // Anal. Biochem. 2002. Vol. 302. P. 52-59.

[8] Федоров А.А., Сочивко Д.Г., Варламов Д.А., Курочкин B.E. // ЖТФ. 2018. Т. 88. Вып. 10. С. 1478-1481. [Fedorov A.A., Sochivko D.G., Varlamov D.A., Kurochkin V.E., Petrov R.V. // Tech. Phys. 2019. Vol. 63. P. 1430-1434.]

[9] Сочивко Д.Г., Федоров А.А., Варламов Д.А. Курочкин В.Е., Петров Р.В. // ДАН. 2016. Т. 466. С. 109-113. [Sochivko D.G., Fedorov A.A., Varlamov D.A., Kurochkin V.E., Petrov R.V. // Doklady Biochemistry and Biophysics 2016. Vol. 466. P. 13-16.]

[10] Сочивко Д.Г., Федоров А.А., Алексеев Я.И., Курочкин В.Е., Дубина М.В. // ДАН. 2017. Т. 472. С. 726-729. [Sochivko D.G., Fedorov A.A., Varlamov D.A., Kurochkin V.E., Petrov R.V. // Doklady Biochemistry and Biophysics. 2017. Vol. 472. P. 77-80.] 